ISSN: 2578-8760

\title{
Self-Joule Heating Activated Mask for Combating COVID-19
}

\author{
Barnali Ghatak ${ }^{1}$; Sanjoy Banerjee ${ }^{2}$; Sk Babar Ali3 ; Nityananda Das ${ }^{4}$; Bipan Tudu ${ }^{*}$; Dipankar Mandal5*; Rajib Bandyopadhyay ${ }^{1,6 *}$ \\ ${ }^{1}$ Department of Instrumentation and Electronics Engineering, Jadavpur University, Kolkata 700106, India \\ ${ }^{2}$ Department of Applied Electronics and Instrumentation Engineering, Future Institute of Engineering and Management, Kolkata \\ 700150, India
}

${ }^{3}$ Department of Electronics and Communication Engineering, Future Institute of Engineering and Management, Kolkata 700150, India

${ }^{4}$ Department of Physics, Jagannath Kishore College, Purulia 723101, West Bengal, India

5 Institute of Nano Science \& Technology (INST), Phase-10, Sector-64, Mohali, Punjab 160062, India

${ }^{6}$ Laboratory of Artificial Sensory Systems, ITMO University, Saint Petersburg, 191002, Russia

\author{
*Corresponding Author(s): Bipan Tudu1, Dipankar \\ Mandal $^{2}$, Rajib Bandyopadhyay ${ }^{3}$ \\ ${ }^{1}$ Department of Instrumentation and Electronics Engi- \\ neering, Jadavpur University, Kolkata 700106, India \\ ${ }^{2}$ Institute of Nano Science \& Technology (INST), Phase- \\ 10, Sector-64, Mohali, Punjab 160062, India \\ ${ }^{3}$ Laboratory of Artificial Sensory Systems, ITMO Univer- \\ sity, Saint Petersburg, 191002, Russia \\ Email: bipantudu@gmail.com,dmandal@inst.ac.in, \\ bandyopadhyay.rajib@gmail.com
}

Received: Apr 30, 2020

Accepted: Jun 05, 2020

Published Online: Jun 10, 2020

Journal: Journal of Nanomedicine

Publisher: MedDocs Publishers LLC

Online edition: http://meddocsonline.org/

DOI: http://doi.org/10.33582/2578-8760/1027

Copyright: (C) Tudu B, Mandal D, Bandyopadhyay R (2020). This Article is distributed under the terms of Creative Commons Attribution 4.0 International License

\section{Abstract}

As the deadly virus is continuing to infect the entire population of the globe, proper protection against the novel coronavirus has become extremely crucial. The virus is entering through our nostrils casusing extensive damage to the tissues of the lungs and wearing a good mask has become mandatory to the virus attack. In this paper, we present the design of a self-Joule heat activated mask, which can generate static electricity due to friction between the layers of the mask which activate the external layer made with metallic mesh that can kill the virus when it passes through this outer layer. The inner and middle layers are so designed using two materials from the two extreme ends of a triboelectric series, which escalated the chances of producing more static electricity between these two layers. The generated power (in the range of $\mathrm{mJ}$ ) can produce heat if the output leads of troelectric nanogenerator are connected to a suitable conducting mesh arranged in the outermost layer. Interestingly, the functioning of the proposed mask is bidirectional so that the virus-carrying exhaled air gets filtered in the static filter between the first two layers and the virus-containing aerosol can be inactivated in the outer layer. The smart filter is energized internally without any external power supply. Overall, the filtration efficiency $(n m-\mu m)$ of the proposed mask is found to be promising and can be used over a large number of people.

Keywords: COVID-19; Static-electricity; Triboelectric nanogenerator; Self-Joule heating activated mask; Smart filter.

Cite this article: Ghatak B, Banerjee S, Barbar Ali SK, Das N, Tudu B, Mandal D, et al. Self-Joule Heating Activated Mask for Combating COVID-19. J Nanomed. 2020; 3(1): 1027. 


\section{Introduction}

The recent pandemic due to COVID-19 has descended with an extraordinary speed and intensity. It has now become an utmost requirement for every person to wear cloth masks when they are out and about. The transmission of a novel coronavirus is through the respiratory droplets in the form of either bioaerosols or droplets generated directly by patients' exhalation. Few studies envisaged that the aerosols $(<1 \mu \mathrm{m})$ can stay for $8 \mathrm{~h}$ in the environment whereas the SARS-CoV-2 virus is found to stay for $3 \mathrm{~h}$ in the environment [1]. Though the relative effectiveness of different droplet sizes in transmitting the SARS-CoV-2 virus is yet ambiguous, developing an efficient filter for particles is highly demanding.

\section{Proposed Methodology}

The present concept is represented in the appended schematic diagram (Figure 1). We term it as "Self-Joule heating activated mask". The notion here is to utilize the triboelectric properties of polymer materials for providing electrostatic filtration once the infectious particle comes in contact with the target person. The proposed mask comprises of three layers - the first two layers (inner and middle) are made up of two materials from the two extreme ends of a triboelectric series, which will enhance the chances of getting more static electricity due to friction between these two layers. The extreme outer part of the mask is designed to develop the produced electrostatic energy (possible to produce in the range of $\mathrm{mW}$ to $\mathrm{kW}$ [2-6]) by forming an electrically active mesh that might have the capability to destroy the activity of the incoming virus.

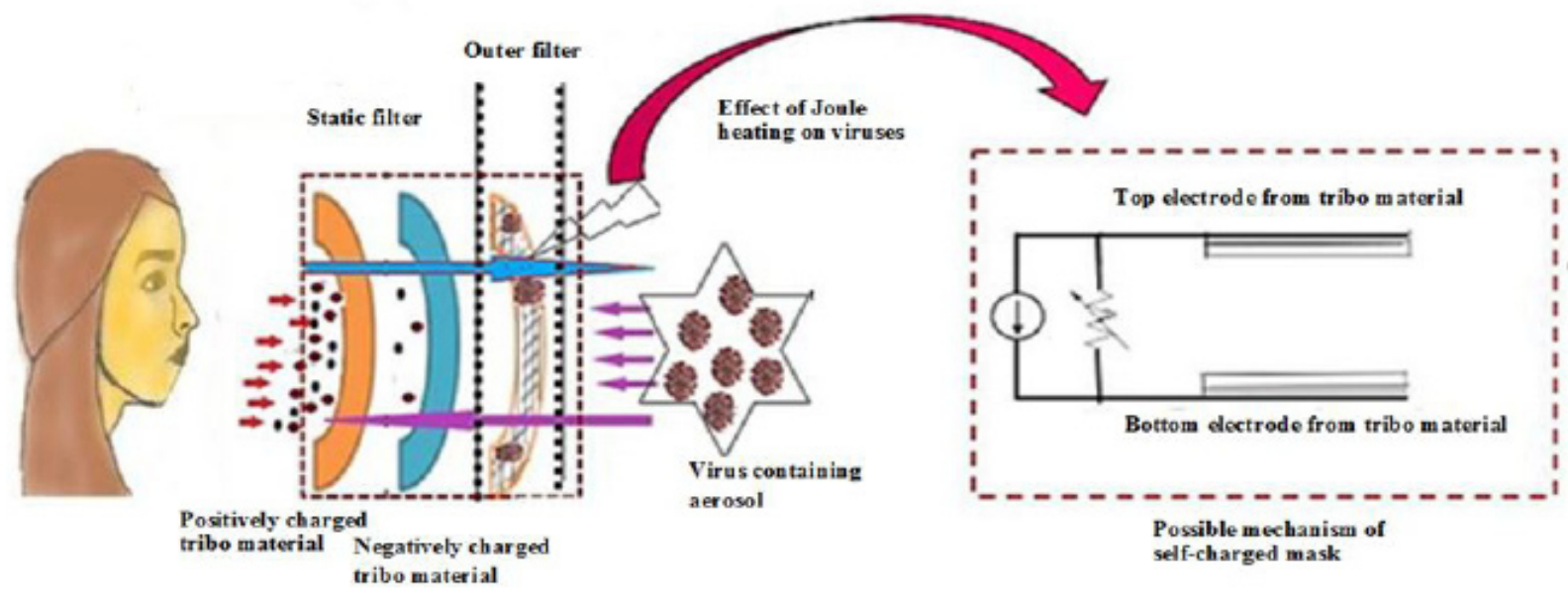

Figure 1: Schematic representation of the possible mechanism of a three-layered 'self-Joule heating activated mask'.

The conceptualized self-chaged mask can be functioned from the both sides. The triboelectric nano-generator would be self-chared i.e. no external power is required to turn it on [1]. The air pressure exerted during breathing is enough to enable the friction between two differently charged materials present in the first two layers of the mask $[7,8]$. Besides, breathing gestures during the speech can increase the output energy density. The generated energy (in the range of $\mathrm{mJ}$ ) is utilized to produce the heat due to the Joule effect if output leads of of the triboelectric materials are connected to a suitable conducting mesh arranged in the outermost layer [9].

The voltage that can be produced by tribolayers due to friction during breathing is expected to be 5 to $10 \mathrm{~V}$. The virus carrying droplets are more or less spherical in shape and have comparatively high resistance $(\sim \mathrm{k} \Omega)$. But as the droplets come in between the conducting mesh, the virus gets deformed and flattened. As a result, the spherical shape deformed into a layer of water (with virus in it), the resistance comes down to 10 to $100 \Omega$. So the produced voltage, current, and heat can be calculated as follows:

Let assume the voltage generated between triboelectric layers $(\mathrm{V})=10 \mathrm{~V}$ and $\mathrm{R}=1000 \Omega$ (assuming the maximum possible resistance of the deformed droplet in the conducting mesh). It give rise the current of $0.01 \mathrm{~A}$.
Thus, the output power $\left(I^{2} R\right)$ would be $~ 100 \mathrm{~mW}$, which is sufficient to warm up and deactivate the viruses with sufficient percentages. If the wearer comes in contact with aninfected person, the incoming viral particles will possibly get inactive while it come to contact to the outer mesh. This phenomenon rendered the activity of any viral entities. In consequence, this effect efficaciously reduced the chances of entering the virus into the inner layers.

Conclusion with key outcomes of the proposed Joule heating activated mask

The salient features of the mask would be the following:

- $\quad$ No external power is required (triboelectric nanogenerator can harvest the mechanical energy from the exhaled air pressure and lips motions during speech or self-talk or sing a song or talking with some-one).

- $\quad$ The proposed technology would be restricting the virus particles to release in the environment through electrostatic adsorption caused by the static charges.

- Utilization of the triboelectric built-in potential through conduction of electrical current in the outermesh.

- $\quad$ The conceptualized outer filter is expected to exhibit a broad action range of particle size (nanometers to microns) with high removal efficiency due to electrostatic filtration. 
- Another essence of the present concept is to incorporate nanoparticles coated printed multilayer sheets for attaining higher filtration efficiency of the proposed mask.

- $\quad$ Cost-effectiveness (since the material cost would be very low, for example, ethyl cellulose-containing inner layer, polytetrafluoroethylene/Teflon contained middle layer, woven /net-based outer layer) would be one of the prime features.

\section{Declaration}

B. Ghatak, S. Banerjee, and SK B. Ali are contributed equally. Prof. B. Tudu, Dr. D. Mandal, and Prof. R. Bandyopadhyay are the corresponding authors.

\section{References}

1. Konda A, Prakash A, Moss GA, Grant GD, Guha S. Aerosol Filtration Efficiency of Common Fabrics Used in Respiratory Cloth Masks. ACS Nano. 2020; 14: 6339-6347.

2. Wang Y, Yang Y, Wang ZL. Triboelectric Nanogenerators as Flexible Power Sources. Npj Flexible Electronics. 2017; 1.

3. Chun S, Choi Y, Son W, Jung J, Lee S, Kim HS, Pang C, Park W, Kim JK. High-Output and Bending-Tolerant Triboelectric Nanogen- erator Based on an Interlocked Array of Surface-Functionalized Indium Tin Oxide Nanohelixes. ACS Energy Lett. 2019; 4, 1748175.

4. Song P, Yang G, Lang T, Yong KT. Nanogenerators for Wearable Bioelectronics and Biodevices, J. Phys. D: Appl. Phys. 2018; 52, 023002.

5. Seo B, Cha Y, Kim S, Choi W. Rational Design for Optimizing Hybrid Thermo-triboelectric Generators Targeting Human Activities. ACS Energy Lett. 2019, 4: 2069-2074.

6. Wang ZL. Triboelectric Nanogenerator (TENG)-Sparking an Energy and Sensor Revolution. Adv. Energy Mater. 2020: 2000137.

7. Wang $M$, Zhang J, Tang $Y$, Li J, Zhang B, Liang E, Mao Y, Wang X. Air-Flow-Driven Triboelectric Nanogenerators for Self-Powered Real-Time Respiratory Monitoring, ACS Nano 2018: 12; 61566162.

8. Ouyang H, Liu Z, Li N, Shi B, Zou Y, Xie F, Ma Y, Li Z, Li H, Zheng Q, Qu X, Fan Y, Wang ZL, Zhang H, Li Z. Symbiotic Cardiac Pacemaker. Nat. Comm. 2019: 10.

9. Heating Effect of Current: Physics of Joule Heating. Jangid, P. Kindle Ed. 2017. 